\title{
UNIQUENESS OF MEROMORPHIC FUNCTIONS THAT SHARE THREE VALUES
}

\author{
By SHOU-ZHEN Ye
}

\section{Introduction and Main Results.}

Let $f$ and $g$ be two nonconstant meromorphic functions in the complex plane. If $f$ and $g$ have the same a-points with the same multiplicities, we say $f$ and $g$ share the value a $C M$. (see [1]). It is assumed that the reader is familiar with the notations of the Nevanlinna Theory (see, for example, [2]). Let $E$ denote any set of finite linear measure of $0<r<\infty$. The notation $S(r, f)$ denotes any quantity satisfying

$$
S(r, f)=o(T(r, f)) \quad(r \rightarrow \infty, r \notin E) .
$$

H. Ueda proved the following theorem.

THEOREM A (see [3]). Let $f$ and $g$ be two distinct nonconstant entire functions such that $f$ and $g$ share 0,1 CM., and let a be a finite complex number, and $a \neq 0,1$. If $a$ is lacunary for $f$, then $1-a$ is lacunary for $g$, and

$$
(f-a)(g+a-1) \equiv a(1-a) .
$$

In [4] $Y i$ Hong-Xun proved more generally the following theorem.

THEOREM B. Let $f$ and $g$ be two distinct nonconstant entire functions such that $f$ and $g$ share 0, 1 CM., and let $a$ be a finite complex number, and $a \neq 0,1$. If $\delta(a, f)>1 / 3$, then $a$ and $1-a$ are Picard exceptional values of $f$ and $g$ respectively, and

$$
(f-a)(g+a-1) \equiv a(1-a) .
$$

In this paper we extend the above theorems to meromorphic functions, and, in fact, prove the following theorem.

THEOREM 1. Let $f$ and $g$ be two distinct nonconstant meromorphic functions such that $f$ and $g$ share $0,1, \infty C M$., and let $a$ be a finite complex number, and $a \neq 0,1$. If

Received July 6, 1991; Revised November 5, 1991. 


$$
\delta(a, f)+\delta(\infty, f)>\frac{4}{3},
$$

then $a$ and $1-a$ are Picard exceptional values of $f$ and $g$ respectively, and also $\infty$ is so, and

$$
(f-a)(g+a-1) \equiv a(1-a) .
$$

In place of Theorem 1, we prove more generally the following theorem which is a generalization of Theorem A, Theorem B and Theorem 1.

THEOREM 2. Let $f$ and $g$ be two distinct nonconstant meromorphic functions such that $f$ and $g$ share $0,1, \infty C M$., and let $a_{1}, a_{2}, \cdots, a_{p}$ be $p(\geqq 1)$ distınct finite complex numbers, and $a_{\imath} \neq 0,1(i=1,2, \cdots, p)$. If

$$
\sum_{\imath=1}^{p} \delta\left(a_{\imath}, f\right)+\delta(\infty, f)>\frac{2(p+1)}{p+2},
$$

then there exists one and only one $a_{3}$ in $a_{1}, a_{2}, \cdots, a_{p}$ such that $a_{3}$ and $1-a_{3}$ are Picard exceptional values of $f$ and $g$ respectively, and also $\infty$ is so, and

$$
\left(f-a_{\jmath}\right)\left(g+a_{\jmath}-1\right) \equiv a_{j}\left(1-a_{\jmath}\right) .
$$

\section{Some Lemmas.}

The following lemmas will be needed in the proof of our theorems.

LEMMA 1. Let $f$ and $g$ be two distinct nonconstant meromorphic functions such that $f$ and $g$ share $0,1, \infty C M$., then

$$
\begin{gathered}
f=\frac{1-e^{\beta}}{1-e^{\beta-\alpha}}, \\
f-1=\frac{e^{\beta-\alpha}\left(1-e^{\alpha}\right)}{1-e^{\beta-\alpha}},
\end{gathered}
$$

where $\alpha$ and $\beta$ are entire functions and $e^{\alpha} \not \equiv 1, e^{\beta} \not \equiv 1, e^{\beta-\alpha} \not \equiv 1$ and

$$
\begin{aligned}
& T\left(r, e^{\alpha}\right)=0(T(r, f)) \quad(r \notin E), \\
& T\left(r, e^{\beta}\right)=0(T(r, f)) \quad(r \notin E) .
\end{aligned}
$$

Proof. By assumption, we have

and

$$
f=g e^{\alpha}
$$

$$
f-1=(g-1) e^{\beta},
$$

where both $e^{\alpha}$ and $e^{\beta}$ are entire functions, and 


$$
e^{\alpha} \not \equiv 1, \quad e^{\beta} \not \equiv 1, \quad e^{\beta-\alpha} \not \equiv 1 .
$$

It follows from (4) and (5) that (2) and (3) hold.

By Nevanlinna's second fundamental theorem, we obtain

$$
T(r, g)<N\left(r, \frac{1}{g}\right)+N\left(r, \frac{1}{g-1}\right)+N(r, g)+S(r, g)<3 T(r, f)+S(r, g) .
$$

Hence

$$
T(r, g)<(3+o(1)) T(r, f) \quad(r \notin E) .
$$

It follows from Nevanlinna's first fundamental theorem that

$$
\left.T\left(r, e^{\alpha}\right) \leqq T(r, f)+T\left(r, \frac{1}{g}\right)<(4+o(1)) T(r, f) \quad r \notin E\right)
$$

and

$$
T\left(r, e^{\beta}\right) \leqq T(r, f-1)+T\left(r, \frac{1}{g-1}\right)<(4+o(1)) T(r, f) \quad(r \notin E) .
$$

This completes the proof of Lemma 1 .

LEMMA 2 (see [5]). Let $f_{i}(i=1,2, \cdots, n)$ be $n$ linearly independent meromorphic functions satisfying

$$
\sum_{i=1}^{n} f_{i} \equiv 1,
$$

then for $\jmath=1,2, \cdots, n$ we have

$$
\begin{aligned}
T\left(r, f_{j}\right)< & \sum_{\imath=1}^{n} N\left(r, \frac{1}{f_{\imath}}\right)+N\left(r, f_{j}\right)+N(r, D)-\sum_{\imath=1}^{n} N\left(r, f_{\imath}\right)-N\left(r, \frac{1}{D}\right) \\
& +0\left(\log r+\log T_{n}(r)\right) \quad(r \notin E),
\end{aligned}
$$

where $D$ denotes the Wronskian

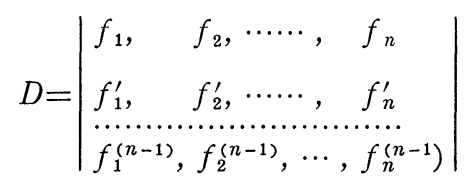

and $T_{n}(r)$ denotes the maximum of $T\left(r, f_{\imath}\right)(i=1,2, \cdots, n)$.

LEMMA 3. Let $b$ be a finite complex number, and $b \neq 0,1$. Suppose that $f$ and $g$ are two distinct nonconstant meromorphic functions such that $f$ and $g$ share $0,1, \infty C M$.. Using the notations of Lemma 1 , let $f_{1}=(1 / 1-b)(f-b)\left(1-e^{\beta-\alpha}\right)$, $f_{2}=(1 / 1-b) e^{\beta}, f_{3}=(-b / 1-b) e^{\beta-\alpha}$. If the $f_{i}(i=1,2,3)$ are linearly independent, then

$$
N\left(r, \frac{1}{f}\right)<N\left(r, \frac{1}{f-b}\right)+S(r, f)
$$




$$
N\left(r, \frac{1}{f-1}\right)<N\left(r, \frac{1}{f-b}\right)+S(r, f) .
$$

Proof. By assumption, from Lemma 1 we easily see that all $f_{i}(i=1,2,3)$ are entire functions, and

$$
\begin{aligned}
& \sum_{i=1}^{3} f_{i} \equiv 1, \\
& T_{3}(r)=0(T(r, f)),
\end{aligned}
$$

where $T_{3}(r)$ denotes the maximum of $T\left(r, f_{\imath}\right)(i=1,2,3)$.

Applying Lemma 2 to functions $f_{\imath}(i=1,2,3)$ we obtain

$$
T\left(r, e^{\beta}\right)<N\left(r, \frac{1}{f-b}\right)+N\left(r, \frac{1}{e^{\beta-a}-1}\right)-N(r, f)+S(r, f) .
$$

Note that $e^{\beta} \neq$ const. and (2), then we have

$$
\begin{aligned}
N\left(r, \frac{1}{f}\right) & =N\left(r, \frac{1}{e^{\beta}-1}\right)-N\left(r, \frac{1}{e^{\beta-\alpha}-1}\right)+N(r, f) \\
& =T\left(r, e^{\beta}\right)-N\left(r, \frac{1}{e^{\beta-\alpha}-1}\right)+N(r, f)+S(r, f) \\
& <N\left(r, \frac{1}{f-b}\right)+S(r, f) .
\end{aligned}
$$

Let us put

$$
g_{1}=\frac{1}{b} e^{\alpha-\beta}(f-b)\left(1-e^{\beta-a}\right), \quad g_{2}=\frac{1}{b} e^{\alpha}, \quad g_{3}=-\frac{1-b}{b} e^{\alpha-\beta}, \quad \text { then } \sum_{i=1}^{3} g_{i} \equiv 1 .
$$

Assume that the $g_{i}(\imath=1,2,3)$ are linearly dependent, then there would be constants $d_{i}(\imath=1,2,3)$ which can't all equal zero, such that

$$
d_{1} g_{1}+d_{2} g_{2}+d_{3} g_{3}=0 \text {. }
$$

Multiplying the above equation by $(b / 1-b) e^{\beta-\alpha}$, and noting that $\sum_{i=1}^{3} f_{i} \equiv 1$, we obtain

$$
\left(d_{1}-d_{3}\right) f_{1}+\left(d_{2}-d_{3}\right) f_{2}-d_{3} f_{3}=0 .
$$

Since $d_{1}-d_{3}$ and $d_{2}-d_{3}$ can't all equal zero, hence the $f_{i}(\imath=1,2,3)$ are also linearly dependent, contrary to the above assumption that the $f_{\imath}(\imath=1,2,3)$ are linearly independent. So the $g_{\imath}(\imath=1,2,3)$ must also be linearly independent. Noting that $e^{\alpha} \not \equiv$ const. and (3), in a similar manner, we can prove that

$$
N\left(r, \frac{1}{f-1}\right)<N\left(r, \frac{1}{f-b}\right)+S(r, f) .
$$

This completes the proof of Lemma 3 . 
By Nevanlinna's second fundamental theorem, we can easily prove the following lemma.

LEMMA 4. Let $f$ and $g$ be two nonconstant meromorphic functions, and let $c_{1}, c_{2}$ and $c_{3}$ be three nonzero constants. If

then

$$
c_{1} f+c_{2} g \equiv c_{3},
$$

$$
T(r, f)<\bar{N}\left(r, \frac{1}{f}\right)+\bar{N}\left(r, \frac{1}{g}\right)+\bar{N}(r, f)+S(r, f) .
$$

\section{Proof of Theorem 2.}

In the following, we shall use the notations of Lemma 1 and Lemma 3.

Suppose that the $f_{i}(i=1,2,3)$ are linearly independent for any $b=a_{\imath}(i=1$, $2, \cdots, p)$. By Lemma 3

$$
N\left(r, \frac{1}{f}\right)<N\left(r, \frac{1}{f-a_{\imath}}\right)+S(r, f) \quad(i=1,2, \cdots, p)
$$

and

$$
N\left(r, \frac{1}{f-1}\right)<N\left(r, \frac{1}{f-a_{\imath}}\right)+S(r, f) \quad(i=1,2, \cdots, p) .
$$

Hence we have

$$
N\left(r, \frac{1}{f}\right)<\frac{1}{p} \sum_{\imath=1}^{p} N\left(r, \frac{1}{f-a_{\imath}}\right)+S(r, f)
$$

and

$$
N\left(r, \frac{1}{f-1}\right)<\frac{1}{p} \sum_{\imath=1}^{p} N\left(r, \frac{1}{f-a_{\imath}}\right)+S(r, f) .
$$

By Nevanlinna's second fundamental theorem

$$
\begin{aligned}
(p+1) T(r, f) & <N\left(r, \frac{1}{f}\right)+N\left(r, \frac{1}{f-1}\right)+N(r, f)+\sum_{\imath=1}^{p} N\left(r, \frac{1}{f-a_{\imath}}\right)+S(r, f) \\
& <\left(1+\frac{2}{p}\right)\left(\sum_{\imath=1}^{p} N\left(r, \frac{1}{f-a_{\imath}}\right)+N(r, f)\right)+S(r, f) \\
& \leqq \frac{p+2}{p}\left\{p+1-\left(\sum_{\imath=1}^{p} \delta\left(a_{\imath}, f\right)+\delta(\infty, f)\right)\right\} T(r, f)+S(r, f) .
\end{aligned}
$$

Since

$$
\frac{p+2}{p}\left\{p+1-\left(\sum_{i=1}^{p} \delta\left(a_{i}, f\right)+\delta(\infty, f)\right)\right\}<p+1,
$$

so (6) is a contradiction. Hence the $f_{i}(i=1,2,3)$ are linearly dependent for at 
least one of the $a_{i}(i=1,2, \cdots, p)$, say for $b=a_{1}$. Thus for the fixed value $b=$ $a_{1}$ there would be three constants $\left(c_{1}, c_{2}, c_{3}\right) \neq(0,0,0)$ such that

$$
c_{1} f_{1}+c_{2} f_{2}+c_{3} f_{3}=0 \text {. }
$$

If $c_{1}=0$, from (7) we have $c_{2} \neq 0, c_{3} \neq 0$ and $e^{\alpha} \equiv k_{1}$, where $k_{1}=\left(b c_{3} / c_{2}\right)$ clearly is a constant which depends on $b=a_{1}$, and $k_{1} \neq 0,1$. Then we have $f-1 \neq 0$ by (3). Hence by Nevanlinna's second fundamental theorem

$$
\begin{aligned}
p T(r, f) & <N\left(r, \frac{1}{f-1}\right)+N(r, f)+\sum_{\imath=1}^{p} N\left(r, \frac{1}{f-a_{\imath}}\right)+S(r, f) \\
& =N(r, f)+\sum_{\imath=1}^{p} N\left(r, \frac{1}{f-a_{\imath}}\right)+S(r, f) .
\end{aligned}
$$

So that

$$
\sum_{\imath=1}^{p} \delta\left(a_{\imath}, f\right)+\delta(\infty, f) \leqq 1
$$

which contradicts the condition (1) of the theorem. Thus $c_{1} \neq 0$.

In the following, suppose that $c_{1} \neq 0$. From (7) we get

$$
f_{1}=-\frac{c_{2}}{c_{1}} f_{2}-\frac{c_{3}}{c_{1}} f_{3} \text {. }
$$

Hence

$$
\left(1-\frac{c_{2}}{c_{1}}\right) f_{2}+\left(1-\frac{c_{3}}{c_{1}}\right) f_{3} \equiv 1
$$

We shall discuss the following four cases:

a) Assume $1-\left(c_{2} / c_{1}\right) \neq 0$ and $1-\left(c_{3} / c_{1}\right) \neq 0$.

If both $e^{\beta}$ and $e^{\beta-\alpha}$ are nonconstants, then, by Lemma 4 , we obtain

$$
T\left(r, e^{\beta}\right)<\bar{N}\left(r, \frac{1}{e^{\beta}}\right)+\bar{N}\left(r, \frac{1}{e^{\beta-\alpha}}\right)+\bar{N}\left(r, e^{\beta}\right)+S\left(r, e^{\beta}\right)=o\left(T\left(r, e^{\beta}\right)\right) \quad(r \notin E),
$$

which is impossible. Thus at least one of $e^{\beta}=(1-b) f_{2}$ and $e^{\beta-\alpha}=-(1-b / b) f_{3}$ would equal a constant, so that both of them would be so by (8). Hence $f$ and $g$ are reduced to constants, which is a contradiction. Therefore this case is impossible.

b) Assume $1-\left(c_{2} / c_{1}\right)=0$ and $1-\left(c_{3} / c_{1}\right) \neq 0$.

Clearly $e^{\beta-\alpha} \equiv k_{2}$, where $k_{2}$ is a constant which depends on $b=a_{1}$, and $k_{2}$ $\neq 0,1$.

Then we have $f=\left(1-e^{\beta} / 1-k_{2}\right)$ by (2). For any complex number $c$ we obtain

$$
f-c=\frac{1}{1-k_{2}}\left\{1-c\left(1-k_{2}\right)-e^{\beta}\right\}
$$


If $1-c\left(1-k_{2}\right) \neq 0$, then

$$
\delta(c, f)=0 .
$$

Since $\delta(\infty, f)=1$, it follows from (1)

$$
\sum_{\imath=1}^{p} \delta\left(a_{\imath}, f\right)>\frac{p}{p+2} .
$$

Hence there exists one and only one $a_{\jmath}$ in $a_{1}, a_{2}, \cdots, a_{p}$ such that

Thus

$$
1-a_{j}\left(1-k_{2}\right)=0 \text {. }
$$

$$
f=a_{j}\left(1-e^{\beta}\right), \quad g=\left(1-a_{\jmath}\right)\left(1-e^{-\beta}\right) .
$$

Consequently

$$
\left(f-a_{\jmath}\right)\left(g+a_{j}-1\right) \equiv a_{\jmath}\left(1-a_{\jmath}\right),
$$

and in this case $a$, and $1-a$, are Picard exceptional values of $f$ and $g$ respectively, and also $\infty$ is so.

c) Assume $1-\left(c_{2} / c_{1}\right) \neq 0$ and $1-\left(c_{3} / c_{1}\right)=0$.

Clearly $e^{\beta} \equiv$ const.. As the same as the case when $c_{1}=0$, this case is impossible too.

In fact, then we have $f \neq 0$ and

$$
\sum_{\imath=1}^{p} \delta\left(a_{\imath}, f\right)+\delta(\infty, f) \leqq 1
$$

by Nevanlinna's second fundamental theorem.

d) Assume $1-\left(c_{2} / c_{1}\right)=0$ and $1-\left(c_{3} / c_{1}\right)=0$.

Clearly we have $c_{1}=c_{2}=c_{3}$, which contradicts $\sum_{i=1}^{3} f_{i} \equiv 1$. Thus this case is also impossible.

Summarize the above, we conclude that under the hypotheses of the theorem, there exists one and only one $a_{3}$ in $a_{1}, a_{2}, \cdots, a_{p}$ such that $a_{3}$ and $1-a$, are Picard exceptional values of $f$ and $g$ respectively, and also $\infty$ is so, and

$$
\left(f-a_{\jmath}\right)\left(g+a_{j}-1\right) \equiv a_{j}\left(1-a_{\jmath}\right) .
$$

This completes the proof of Theorem 2 .

Acknowledgement. I am grateful to the referee for valuable comments.

\section{REFERENCES}

[1] G.G. Gundersen, Meromorphic functions that share three or four values, J. London Math. Soc., (2), 20 (1979), 457-466.

[2] W. K. Hayman, Meromorphic functions, Clarendon Press. Oxford, 1964.

[3] H. UEDA, Unicity theorems for meromorphic or entire functions, Kodai Math. J., 3 (1980), 457-471. 
[4] Hong-Xun YI, Meromorphic functions that share three values, Chin. Ann. Math., 9A (1988), 434-439.

[5] F. Gross, Factorization of meromorphic functions, U.S. Govt. Printing Office Publication, Washington D.C., 1972.

Department of Mathematics

Wenzhou Teacher's College

Wenzhou, ZhejIANG, 325000

P.R. OF CHINA 